\title{
Experiencias en la captura de los gases de combustión de la cascarilla de arroz con soluciones alcalinas
}

\section{Experiences in the capture of combustion gases of rice husk with alkaline solutions}

\author{
Abelardo Prada - Matiz¹, Caroll E. Cortés - Castillo² \\ ${ }^{1}$ Ing. Qco, PhD, Universidad de los Llanos. \\ ${ }^{2}$ Qco. Esp. Gestión ambiental, Universidad de los Llanos. \\ Grupo de Investigación Gestión Ambiental Sostenible - GiGAS. Universidad de los Llanos \\ E-mail: aprada@unillanos.edu.co
}

Recibido: Abril 15 de 2010. Aceptado: Abril 7 de 2011

\section{RESUMEN}

El presente trabajo se orienta al estudio de los procesos de captura de los gases de combustión de la cascarilla de arroz con soluciones alcalinas de hidróxido de sodio $(\mathrm{NaOH})$, hidróxido de calcio $\mathrm{Ca}(\mathrm{OH})_{2} \mathrm{e}$ hidróxido de Potasio $(\mathrm{KOH})$ y su posterior transformación en carbonato de calcio $\left(\mathrm{CaCO}_{3}\right)$, sustancia útil en labores agrícolas, por adición de cloruro de calcio $\left(\mathrm{CaCl}_{2}\right)$ en solución acuosa. Se determinaron las características básicas del proceso de captura de los gases: tiempo de reacción, $\mathrm{pH}$, la masa y el rendimiento del carbonato de calcio $\left(\mathrm{CaCO}_{3}\right)$ obtenido.

Palabras clave: Combustión, cascarilla de arroz, captura de dióxido de carbono, carbonato de calcio.

\section{SUMMARY}

The present work is aimed at studying the processes of capture of combustion gases of rice husk with alkaline solutions of sodium hydroxide $(\mathrm{NaOH})$ and potassium hydroxide $(\mathrm{KOH})$ and its subsequent transformation into calcium carbonate $\left(\mathrm{CaCO}_{3}\right)$, a substance useful in agriculture, by adding calcium chloride $\left(\mathrm{CaCl}_{2}\right)$ in aqueous solution. Was determined the basic characteristics of the process of capture of combustion gases such as reaction time, $\mathrm{pH}$, mass and performance of calcium carbonate $\left(\mathrm{CaCO}_{3}\right)$ obtained.

Key words: Combustion, rice husk, carbon dioxide capture, calcium carbonate.

\section{INTRODUCCIÓN}

De conformidad con el histórico estadístico de Fedearroz, en la región de los Llanos se ha producido, en los últimos diez años, un promedio de 773 mil Ton de arroz/año, generando un total de 155 mil Ton/año de cascarilla de arroz como residuo año tras año (Fedearroz, 2011). Por tanto, 
la cascarilla de arroz se convierte en uno de los desechos más importantes de la producción agrícola de la Orinoquia colombiana, con la particularidad, que, en condiciones naturales, se descompone lentamente, haciendo de su disposición, un problema ambiental considerable, dentro de los que enfrenta la producción arrocera de la zona.

La cascarilla de arroz es de consistencia quebradiza, abrasiva y su color varía del pardo rojizo al púrpura oscuro. Su densidad es baja, por lo cual al apilarse ocupa grandes espacios. El peso específico es de $125 \mathrm{~kg} / \mathrm{m}^{3}$, es decir, 1 tonelada ocupa un espacio de $8 \mathrm{~m}^{3}$ a granel (Varón, 2005). Los principales componentes de la cascarilla de arroz son la celulosa y la hemicelulosa (50\%), además de lignina (26\%) y componentes orgánicos como aceites y proteínas (4\%). Aproximadamente, el $20 \%$ restante incluye diferentes sustancias de carácter inorgánico como $\mathrm{SiO} 2, \mathrm{Al} 2 \mathrm{O} 3, \mathrm{~K} 2 \mathrm{O}$, $\mathrm{Na} 2 \mathrm{O}, \mathrm{MgO}, \mathrm{CaO}, \mathrm{Fe} 2 \mathrm{O} 3, \mathrm{MnO}, \mathrm{P} 2 \mathrm{O} 5$. (Martínez et al, 2010).

El poder calorífico de la cascarilla alcanza 3.281,6 $\mathrm{Kcal} / \mathrm{kg}$. La temperatura máxima, al someterla a combustión, varía de acuerdo con su condición: $970^{\circ} \mathrm{C}$ (seca), $650^{\circ} \mathrm{C}$ (con algún grado de humedad) y hasta los $1000^{\circ} \mathrm{C}$ (mezclada con combustible) (Varón, 2005). La cascarilla de arroz al quemarse genera del $14-25 \%$ de cenizas ricas en sílice (94.5\%) dependiendo de la variedad de arroz, clima y suelo de la región (Krishnarao et al. 2001)

Las experiencias más relevantes de aprovechamiento de la cascarilla en ensayos de descomposición de la cascarilla de arroz por medios biológicos o con la ayuda de reactivos químicos van desde la obtención de etanol, concreto, cemento, cerámicas, carboximetilcelulosa de sodio; dióxido de $\mathrm{SiO}_{2}$, nitruro de silicio; furfural pasando por el uso como material de relleno para uso agropecuario (cama en avicultura, porcicultura y en transporte de ganado), como fuente energética (ladrilleras, secado de arroz y cereales), como aditivos para obtener otros materiales (Producción de aglomerados - tableros y material aislante en construcción) y en otros casos, por tratamiento térmico previo, se le da uso como sustrato en el cultivos hidropónicos de flores, producción de cenizas para cultivo de frutas y también para trabajar como coadyuvantes en los procesos de captura de dióxido de carbono $\mathrm{CO}_{2}$ en sorbentes cálcicos (López, 2006, Arcos 2007, Li et al 2009)

Las experiencias realizadas en cada uno de estos campos han tenido que superar obstáculos importantes, puesto que una parte considerable de los productos obtenidos no han logrado el uso proyectado, en razón a que las investigaciones en su gran mayoría, no han llegado a la etapa de implementación a escala, en consecuencia, no se dispone de información real del total de cascarilla de arroz consumida en cada alternativa, ni se han realizado estudios de viabilidad técnica, económica y ambiental (Forero, 2001).

Las dificultades encontradas están directamente relacionadas con las características de la cascarilla de arroz, tales como el alto contenido de silicio, que la convierte en un material de muy baja degradabilidad. Además, se ha demostrado que muy pocos organismos vivos se nutren de ella (Cortés, 1999).

Por otra parte, en los casos de utilizar la cascarilla como relleno en camas para la producción porcícola o avícola, en los que se logra reducir, de manera sensible, el consumo de agua, puesto que la cama no la exige para la limpieza, no se conocen datos sobre el uso posterior del residuo, después del contacto físico con los animales, su orina y excretas. En cuanto al propósito de adicionar la cascarilla a otros materiales para obtener cementos, concreto, puzolanas o aglomerados, se tropieza con la dificultad de que la cascarilla de arroz es pobre en los elementos requeridos, por ejemplo, para la producción de cemento el contenido óxidos de calcio, aluminio y hierro en la cascarilla es bajo, no mayor al $1 \%$ en cenizas (Flores, 2004) y para las puzolanas y materiales abrasivos - el silicio es insuficiente (Calleja, 1968). De igual manera, el bajo contenido de carbono, hace que la cascarilla se polimerice con mayor dificultad en comparación con materiales celulósicos como el bagazo (Pérez, 1997, Labrada, 
1998). Por tanto, los productos que se han obtenido con base en la adición de cascarilla no han recibido amplio uso, a pesar de ser más livianos que los tradicionales.

En los casos, en los que a la cascarilla se le da tratamiento térmico previo para, a posteriori, aprovecharla de manera definitiva, se diferencian, plenamente, dos posibilidades: el tratamiento térmico anaeróbico y el tratamiento térmico aeróbico.

El tratamiento térmico anaeróbico tiene como objeto conservar el esqueleto hidrocarbonado de la cascarilla, de manera que se obtenga un material poroso que, luego de ser sometido al flujo del vapor de agua o al contacto con reactivos químicos, se convierta en el denominado carbón activado que se puede utilizar en diferentes procesos industriales, producción de alimentos, tratamiento de agua e incluso la separación de sustancias tóxicas de la sangre (López, 2006, Fernández, 2006).

Los carbones activados obtenidos a partir de cascarilla de arroz, por sus características generales y específicas, no son inferiores a los mismos obtenidos de materiales tradicionales (aserrín de pino, huesos, etc.) e incluso superiores a los obtenidos de residuos de la industria azucarera (Prada, 1986; Castellanos, 1984). Sin embargo, la fragilidad física de los carbones de cascarilla hace que con gran facilidad se pulverizan, dificultando su reactivación, condición importante para justificar su producción. Por tanto, se requiere adicionar otros materiales que los hagan consistentes, lo que los encarece y no hace atractiva su obtención. De todas maneras, este podría ser un importante campo por explorar.

El tratamiento térmico aeróbico es fundamento de la quema a cielo abierto o la prequema controlada, orientada a utilizar el producto de la "prequema" en el cultivo de flores (Lopez, 2006). En los dos casos (quema a cielo abierto y prequema controlada) se generan cantidades importantes de dióxido de carbono - $\mathrm{CO}_{2}$ que, al desprenderse, contribuye al incremento de la concentración de este gas en el aire. Por esta razón, mientras los gases de combustión no se capturen, se debe prescindir de la práctica de la combustión a cielo abierto o la prequema, a pesar que la cascarilla no se encuentra dentro de los materiales generadores de dioxinas (Gorachategi, 2001).

La combustión plena tropieza con una dificultad adicional que consiste en que la capacidad calorífica de la cascarilla es muy inferior a la de los materiales combustibles tradicionales (Tardeu, 2003) y no es la más alta entre los materiales de desecho (López, 2006), situación que hace que no se le prefiera al utilizarla como fuente de energía.

Sin embargo, dentro de las aplicaciones adelantadas en búsqueda de soluciones para la disposición final de la cascarilla, la combustión plena ofrece importantes posibilidades, puesto que las cenizas pueden ser aprovechadas en procesos agrícolas, el calor generado - en diferentes procesos que requieren energía, siempre y cuando se capturen y se les dé un uso adecuado a los gases de combustión, dentro de los cuales, se encuentra el dióxido de carbono.

El dióxido de carbono - $\mathrm{CO}_{2}$, es un gas incoloro que se licúa a grandes presiones y bajas temperaturas. Presenta una solubilidad de 0,145 g/100 g de agua. La inhalación produce asfixia y la exposición puede ser peligrosa (Degremont, 1979). Se incluye dentro del grupo de gases y sustancias que generan el efecto invernadero (Hernández, 2001), en particular aquel que se genera por combustión de materiales que contienen carbono.

Dentro de las reacciones típicas del dióxido de carbono se encuentra la reacción con el hidróxido de calcio, en medio acuoso (Jodakov, 1977). En esta reacción se obtiene carbonato de calcio $\left(\mathrm{CaCO}_{3}\right)$. Por tanto, se puede deducir que es posible obtener carbonato de calcio a partir de materiales que por combustión generen dióxido de carbono, como es el caso de la cascarilla de arroz y que el hidróxido actúe como agente de captura de $\mathrm{CO}_{2}$. De igual manera, se comportan los hidróxidos de metales alcalinos, las sales de calcio y de otros metales alcalinotérreos (Melnik, 1975) 
El carbonato de calcio - $\mathrm{CaCO}_{3}$, conocido como caliza, presenta baja solubilidad en agua - $0,013 \mathrm{~g} /$ $100 \mathrm{~g}$ de agua (Merck, 2007), característica que permite separarlo, en forma de precipitado.

El hidróxido de calcio - $\mathrm{Ca}(\mathrm{OH})_{2}$, de igual manera, presenta baja solubilidad en agua, lo que dificulta que en la solución se disponga de la masa estequiométrica requerida para la captura del $\mathrm{CO}_{2}$. Sin embargo, otras sales de calcio presentan alta solubilidad en agua, de manera que se podrían utilizar en los procesos de captura de dióxido de carbono.

A la par, para evitar los efectos del cambio climático, se plantea, como prioridad, la reducción de la descarga de gases de efecto invernadero en la atmósfera y, en consecuencia, lograr que su concentración se reduzca, en razón que ha presentado un incremento de 280 a más de 380 ppm desde el inicio de la época preindustrial hasta la época actual, con el agravante, que cada año aumenta, en cerca de 2 ppm, en consecuencia, según lo reportado (Canadell et al. 2007), el aumento de las emisiones globales de $\mathrm{CO}_{2}$ alcanza el 3,3\% anual, aproximadamente.

Como respuesta a esa preocupación global, han surgido alternativas como la absorción de dióxido de carbono de la atmósfera con soluciones alcalinas, alternativa que ha sido explorada desde 1940 (Spector y Dodge, 1946; Tepe y Dodge, 1943) $\mathrm{y}$, adicionalmente, se ha implementado como tratamiento previo antes de la separación criogénica del aire.

A finales de 1990 Lackner et al., sugirieron el lavado de $\mathrm{CO}_{2}$, a gran escala, captado del aire, técnica que consiste en la depuración por vía húmeda, en la que el $\mathrm{CO}_{2}$ absorbido en una solución de

\section{MATERIALES Y MÉTODOS}

\section{Localización}

Los ensayos de combustión de la cascarilla de arroz, captura de gases de combustión con soluciones hidróxido de sodio -, $\mathrm{NaOH}-$, después de reaccionar produce carbonato de sodio, $\mathrm{Na}_{2} \mathrm{CO}_{3} \mathrm{y}$ un exceso de hidróxido. El éxito de esta operación depende en gran medida de la eficiencia del contacto entre el $\mathrm{CO}_{2}$ e hidróxido de sodio. (Mahmoudkhani, 2009)

Dentro de las propuestas de captura, también aparecen las reacciones del $\mathrm{CO}_{2}$ con sales de aminoácidos y potasio, tales como la la glicina y la taurina en soluciones acuosas, debido a que su naturaleza iónica les da propiedades de baja volatilidad, alta tensión superficial y alta resistencia a la degradación oxidativa. (Vaidya, 2010). Otra propuesta corresponde a la captura de $\mathrm{CO}_{2}$, empleando como sistemas de carbonatación el óxido de calcio $(\mathrm{CaO})$ y el hidróxido de calcio $(\mathrm{Ca}$ $(\mathrm{OH})_{2}$ ) por separado. Se determinó que con el oxido la tasa de carbonatación está asociada a un modelo de cinética que incluye la reacción química intrínseca, seguida por la difusión dentro de la partícula; en el caso de hidróxido, el proceso de carbonatación esta menos impedido por la difusión catalizada por la formación de agua, y su tasa de captura se ajusta a un modelo de cinética química controlada en la interfaz sólida no cubierta por el $\mathrm{CaCO}_{3}$. (Nikulshina et al. 2007)

De lo anterior se deduce la posibilidad de capturar y posteriormente utilizar los gases de combustión de materiales de origen vegetal con diferentes contenidos de carbono que, como la cascarilla de arroz, se utilizan ampliamente en procesos de producción en condiciones de la Orinoquia colombiana, dentro de los cuales la captura con soluciones alcalinas podría presentarse como alternativa. Este trabajo aporta elementos que podrían servir de soporte para construir soluciones en este campo y aprovechar de manera integral la cascarilla de arroz y otros desechos vegetales de la producción agrícola.

alcalinas y la separación del carbonato de calcio obtenido en el proceso, se realizaron en la Granja y 
en los laboratorios de análisis de aguas y de Suelos de la Universidad de los Llanos, sede Barcelona, ubicada en Villavicencio, Meta, kilómetro 7, vía a Puerto López. La composición química del carbonato de calcio obtenido se determinó en el laboratorio de microscopia electrónica de la Universidad Nacional sede Medellín- UNALMED.

En el desarrollo del proyecto se emplearon elementos necesarios para la construcción del sistema de combustión y posterior captura de los gases (Figura 1), compuesto fundamentalmente por materiales reciclables (tanques, canecas y tubos de chatarrería). Se utilizaron, además, los reactivos propios del análisis de aguas y de muestras líquidas de acuerdo con el Standard Methods, así como sustancias para la captura del $\mathrm{CO}_{2}$, tales como: Hidróxido de Sodio ( $\mathrm{NaOH})$, Hidróxido de potasio $(\mathrm{KOH})$, Hidróxido de Calcio -Ca $(\mathrm{OH})_{2}$, y para la posterior precipitación del carbonato de calcio se empleo cloruro de calcio $\left(\mathrm{CaCl}_{2}\right)$, todos estos reactivos de captura y de precipitación eran grado comercial

\section{Secado de la cascarilla}

Para el secado, la cascarilla se extendió en capas delgadas, en un espacio techado y aireado, sobre una superficie plana, cubierta con papel periódico como material adsorbente de la humedad. En los ensayos se utilizaron muestras con humedad inferior al $8 \%$.

\section{Combustión de la cascarilla y captación de dióxido de carbono}

Para la combustión de la cascarilla, se construyó un horno-quemador, en forma de cilindro metálico de cerca de $30 \mathrm{~cm}$. de diámetro y un metro de altura, organizado de la siguiente manera (Figura 1): en la parte intermedia - se ubica la cámara de combustión en la que se deposita la muestra de cascarilla sobre un soporte metálico con agujeros de media pulgada de diámetro; en la parte inferior un espacio para la entrada de aire, $y$, en la parte superior - un tubo metálico de dos pulgadas, para la evacuación de gases de combustión.
La cámara de combustión del horno-quemador dispone, además, de un orificio de dos pulgadas de diámetro en el centro, que posibilita, que al ubicar un cilindro compacto o trozo de madera dentro de él y luego de retirarlo tras la compactación manual del bloque de cascarilla, se forme un corredor para el paso del aire, de abajo hacia arriba.

Una vez completo el montaje se inicia la combustión, induciéndola, en la parte superior de la cámara de combustión, con ACPM y garantizando la ventilación desde la parte inferior del horno. En cada ensayo se utilizan cerca de cuatro kilogramos de cascarilla.

Los gases de combustión se conducen por el tubo de dos pulgadas, de la parte superior del hornoquemador, a una caneca metálica de 25 litros de capacidad que hace las veces de reactor, en la que se deposita la solución alcalina, preparada con 100 gramos de hidróxido de sodio $(\mathrm{NaOH})$ disueltos en 10 litros de agua. En los ensayos preliminares se utilizó hidróxido de potasio $(\mathrm{KOH})$ e Hidróxido de Calcio - $\mathrm{Ca}(\mathrm{OH})$

La reacción se controla por medio de la valoración del $\mathrm{pH}$ de la solución alcalina, al iniciar y al terminar el proceso de combustión. Para lograr contacto entre los gases de combustión y la solución alcalina, en la parte superior del reactor se conecta, en condiciones de hermetismo, un extractor de gases. La solución producto de esta reacción se traslada al laboratorio para la cuantificación de carbonatos, bicarbonatos, alcalinidad, dureza cálcica, dureza total y $\mathrm{pH}$, como parámetros de seguimiento de la reacción de captura de $\mathrm{CO}_{2}$

\section{Obtención de carbonato de calcio}

Sólo fue posible obtener resultados valorables en la separación del carbonato de calcio, obtenido por del contacto de los gases de combustión de la cascarilla de arroz, con las soluciones alcalinas de los hidróxido de Sodio y Potasio ( $\mathrm{NaOH}$ y $\mathrm{KOH}$ ), sin embargo, dado que, los ensayos preliminares no mostraron diferencias sustanciales entre las soluciones de los hidróxido de los dos 
metales alcalinos, por razones de costos, se utilizó preferencialmente hidróxido de sodio.

No fue posible utilizar hidróxido de calcio - $\mathrm{Ca}(\mathrm{OH})_{2}-$ para obtener la solución alcalina, puesto que dada su baja solubilidad en agua, no se pudo disponer de las cantidades estequiométricas requeridas en el experimento.

Se realizaron ensayos con soluciones de cloruro de Calcio $-\mathrm{CaCl}_{2}$ - sal de alta solubilidad en agua, que puede proporcionar iones de calcio a la solución y posibilitar la reacción con los gases de combustión generados. Sin embargo el rendimiento de la obtención del carbonato fue muy bajo, por esta razón se prescindió de uso posterior.
Por tanto, a la solución de hidróxido de sodio, utilizada en la captura de gases de combustión, en el reactor (Figura 1) se agrega la cantidad estequiométrica de cloruro de calcio $\left(\left(\mathrm{CaCl}_{2}\right)\right.$, para la precipitación del carbonato de calcio (Figura 2), luego la muestra se filtra al vacio, se seca en estufa a $45^{\circ} \mathrm{C}$ durante 24 horas, se pesa y se recolecta en bolsas plásticas.

La composición química de los productos de captura de $\mathrm{CO}_{2}$ se determinó mediante microscopia electrónica de barrido realizada con un equipo JEOL JSM 5910LV, previa metalización con oro en un equipo Denton Vaccun Desk en el Laboratorio de Microscopia Avanzada de la Universidad Nacional sede Medellín - UNALMED.

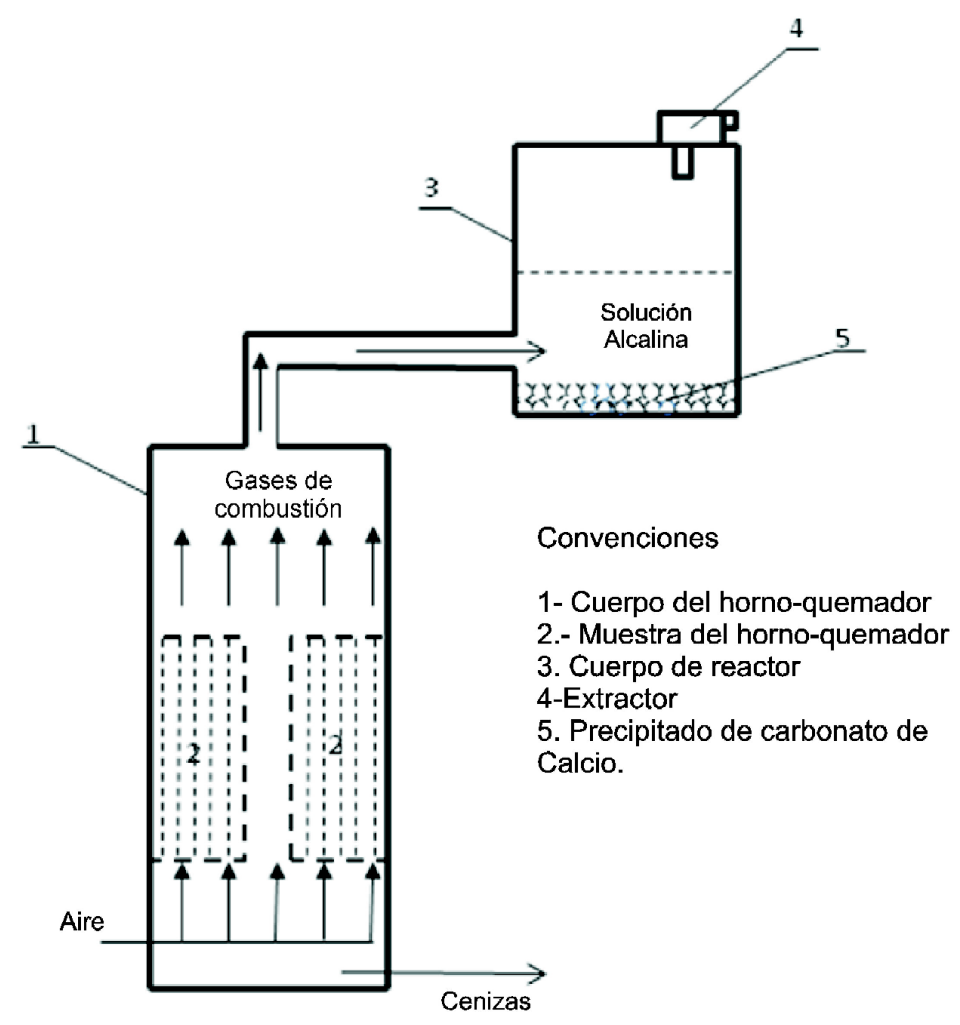

Figura 1. Esquema del horno quemador y del reactor para la captura de los gases de combustión de la cascarilla de arroz 


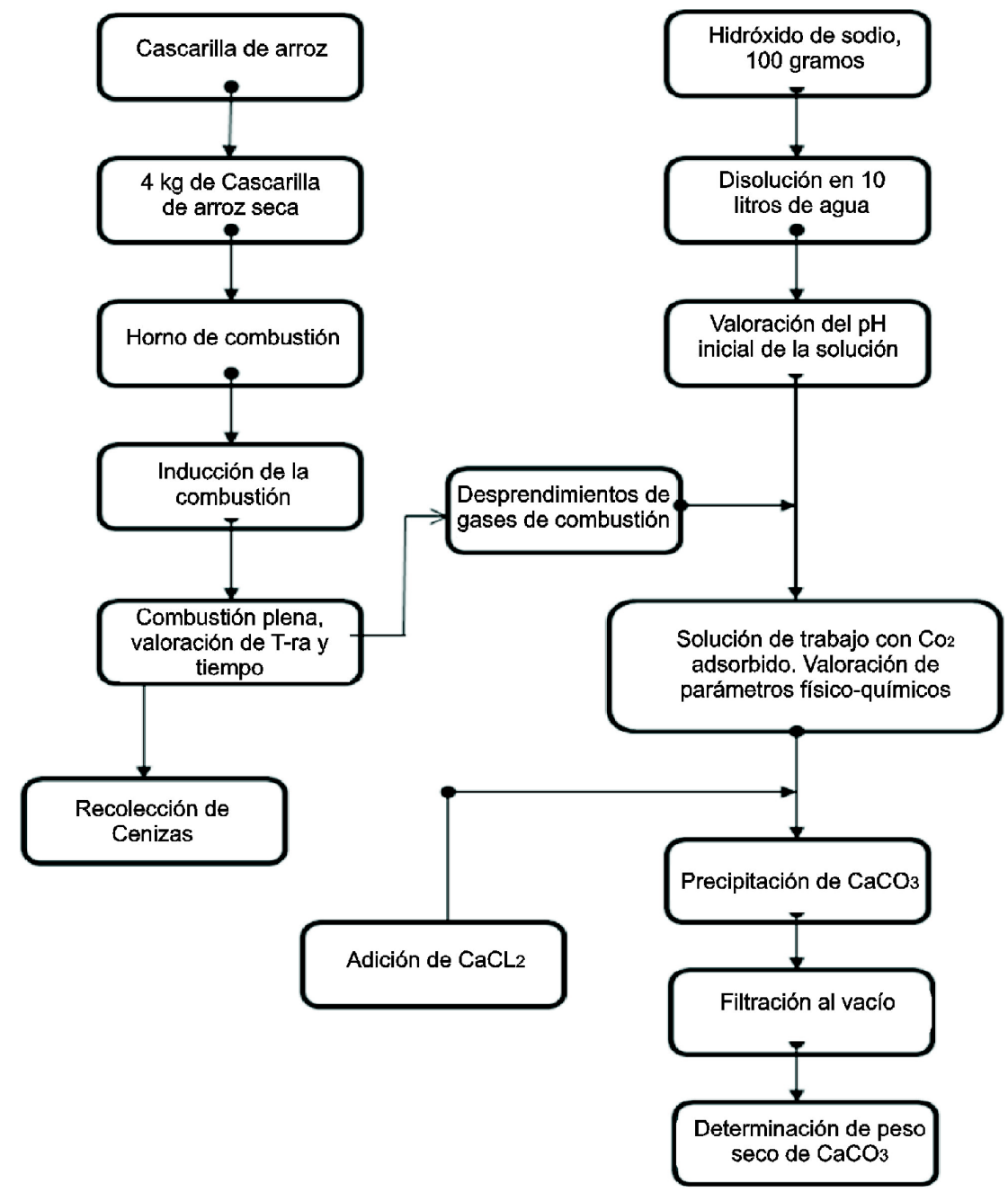

Figura 2. Esquema del proceso de descomposición térmica de la cascarilla de arroz, captura del dióxido de carbono con soluciones alcalinas de hidróxido de sodio y precipitación del carbonato de calcio con cloruro de calcio

\section{ANÁLISIS ESTADÍSTICO}

Los datos obtenidos de los análisis de laboratorio, se procesaron utilizando los métodos de la estadística descriptiva, por medio de la media

\section{RESULTADOS}

Los resultados obtenidos de la captura de gases de combustión de la cascarilla de arroz y separación del carbonato de calcio se exponen de la siguiente manera: verdadera con niveles del $95 \%$ de confianza por medio del cálculo del error estándar, el número de ensayos y el parámetro estadístico T.

En las tablas 2, 3, 4, - los datos de los ensayos en los que se utilizó como solución alcalina 100 gramos de $\mathrm{NaOH}$ en diez litros de agua y en la Figura 3 -los resultados del análisis de la composición química del carbonato calcio obtenido. 
En las tablas, se expone el valor de la característica evaluada en cada ensayo, el promedio, la desviación estándar, el error estándar y el coeficiente de variación, calculados de conformidad con las ecuaciones 2, 3 y 4 . El valor procesado se presenta en forma de la media verdadera.

En las tablas 1, 2 y 3 se incluyen, solamente, los resultados de la captura de los gases de combustión con hidróxido de sodio $(\mathrm{NaOH})$ por las siguientes razones:

La baja solubilidad del hidróxido de calcio - $\mathrm{Ca}(\mathrm{OH})_{2}$, no permite disponer de una masa representativa en el medio de la reacción, por tanto los ensayos con esta sustancia no fue posible realizarlos.

Los ensayos realizados con cloruro de calcio $\left(\mathrm{CaCl}_{2}\right)$, sal de alta solubilidad de agua, que podría ser utilizada en la captura de los gases de combustión, presentaron bajo rendimiento.

Los resultados de los ensayos preliminares con hidróxido de potasio $(\mathrm{KOH})$, no presentaron diferencias significativas con los resultados obtenidos utilizando hidróxido de Sodio $(\mathrm{NaOH})$, por tanto por consideraciones de costos, se seleccionó el hidróxido de sodio como sustancia para la captura de los gases de combustión.

Tabla 1. Tiempo de duración del proceso captura de los gases de combustión de la cascarilla de arroz

\begin{tabular}{cc}
\hline Ensayo & Tiempo (min) \\
\hline 1 & 74 \\
2 & 127 \\
3 & 120 \\
4 & 96 \\
5 & 281 \\
Promedio & 140 \\
Desviación estándar & 82 \\
Error estándar & 94 \\
Coeficiente de variación, $(\%)$ & 58 \\
Media Verdadera, min & $140 \pm 94$ \\
\hline
\end{tabular}

Tabla 2. Valores de pH de la solución de $\mathrm{NaOH}$ en el proceso de captura de los gases de combustión la cascarilla de arroz

\begin{tabular}{ccc}
\hline Ensayo & Valor Inicial del $\mathbf{p H}$ & Valor final del $\mathbf{p H}$ \\
\hline 1 & 12,10 & 8,00 \\
2 & 12,10 & 8,20 \\
3 & 11,90 & 8,20 \\
4 & 12,00 & 8,20 \\
5 & 12,10 & 8,90 \\
Promedio & 12,04 & 8,30 \\
Desviación estándar & 0,09 & 0,35 \\
Error estándar & 0,10 & 0,40 \\
Coeficiente de variación, $\%$ & 0,74 & 4,17 \\
Media Verdadera, unidades & $12,04 \pm 0,10$ & $8,30 \pm 0,40$ \\
\hline
\end{tabular}

La variación de aproximadamente 4,00 unidades de $\mathrm{pH}$ entre la solución inicial de hidróxido de sodio y después del proceso de captura de los gases de combustión, 
Tabla 3. Masa obtenida del carbonato de calcio y porcentaje de rendimiento con relación $100 \mathrm{~g}$ de $\mathrm{NaOH}$ utilizado

\begin{tabular}{lc}
\hline \multicolumn{1}{c}{ Característica } & Valor \\
\hline $\mathrm{P}(\mathrm{ppm})$ & 426.10 \\
$\mathrm{Al}(\mathrm{meq} / 100$ g suelo) & 0.25 \\
$\mathrm{Ca}(\mathrm{meq} / 100 \mathrm{~g}$ suelo) & 17.20 \\
$\mathrm{Mg}(\mathrm{meq} / 100 \mathrm{~g}$ suelo) & 10.80 \\
$\mathrm{~K}$ (meq/100 g suelo) & 4.15 \\
$\mathrm{Na}(\mathrm{meq} / 100 \mathrm{~g}$ suelo) & 0.52 \\
$\mathrm{Cu}(\mathrm{ppm})$ & 10.00 \\
$\mathrm{Fe}(\mathrm{ppm})$ & 10.50 \\
$\mathrm{Mn}(\mathrm{ppm})$ & 537.50 \\
$\mathrm{Zn}(\mathrm{ppm}$ & 67.50 \\
$\mathrm{~B}(\mathrm{ppm})$ & 2.77 \\
$\mathrm{~S}(\mathrm{ppm})$ & 175.80 \\
\hline
\end{tabular}

En general para todos los ensayos se obtuvo en promedio de $66,4 \mathrm{~g} \pm 4,6 \mathrm{~g}$ de Carbonato de calcio, como se puede observar a pesar que el tiempo de combustión y de captura presentaron una alta variabilidad, la cantidad de carbonato obtenido fue prácticamente la misma en los diferentes ensayos.

El carbonato de calcio obtenido producto de la captura de $\mathrm{CO}_{2}$, se llevo sometió a microanálisis de composición por microscopia electrónica de barrido, obteniendo los resultado presentados en la Figura 3.
Estos resultados se repitieron para todas las muestras, los cuales después de procesados confirman que correspondían a carbonato de calcio producto de la captura de $\mathrm{CO}_{2}$ en solución de $\mathrm{NaOH}$ y posterior reacción con $\mathrm{CaCl}_{2}$.

En cuanto a la caracterización de las cenizas obtenidas del proceso de combustión de la cascarilla de arroz (Tabla 4), se encuentra que son ricas en microelementos de importancia en la adecuación de suelos y cuyas capacidades ya se han reportado en diversos trabajos agronómicos

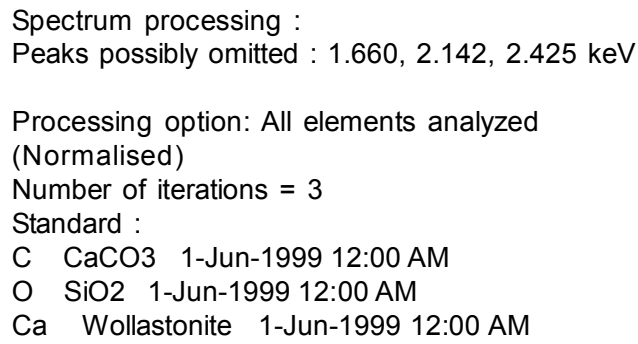

\begin{tabular}{lll}
\hline Element & Weight $\%$ & Atomic $\%$ \\
\hline $\mathrm{C} \mathrm{K}$ & 5.53 & 11.09 \\
$\mathrm{O} \mathrm{K}$ & 35.53 & 53.49 \\
Ca K & 58.94 & 35.42 \\
Totals & 100.00 & \\
& & \\
\hline
\end{tabular}

Laboratorio de Microscopia Electrónica. UNALMED.
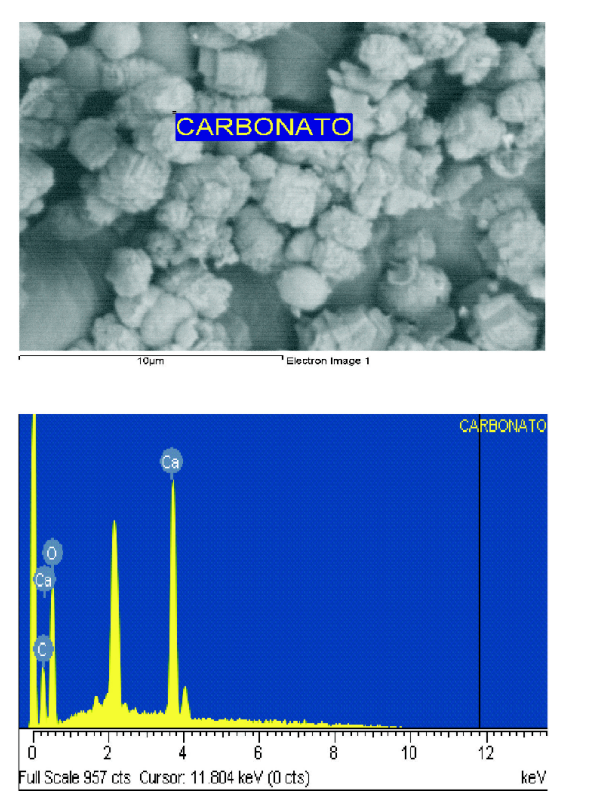

Figura 3. Resultados del análisis de la composición química del carbonato calcio obtenido por descomposición térmica de la cascarilla de arroz y captura de gases con soluciones alcalinas de hidróxido de sodio $(\mathrm{NaOH})$ 
Tabla 4. Composición química de la ceniza de cascarilla de arroz

\begin{tabular}{lc}
\hline \multicolumn{1}{c}{ Característica } & Valor \\
\hline $\mathrm{P}(\mathrm{ppm})$ & 426.10 \\
$\mathrm{Al}(\mathrm{meq} / 100$ g suelo) & 0.25 \\
$\mathrm{Ca}(\mathrm{meq} / 100$ g suelo) & 17.20 \\
$\mathrm{Mg}(\mathrm{meq} / 100$ g suelo) & 10.80 \\
$\mathrm{~K}$ (meq/100 g suelo) & 4.15 \\
$\mathrm{Na}(\mathrm{meq} / 100$ g suelo) & 0.52 \\
$\mathrm{Cu}(\mathrm{ppm})$ & 10.00 \\
$\mathrm{Fe}(\mathrm{ppm})$ & 10.50 \\
$\mathrm{Mn}(\mathrm{ppm})$ & 537.50 \\
$\mathrm{Zn}(\mathrm{ppm}$ & 67.50 \\
$\mathrm{~B}(\mathrm{ppm})$ & 2.77 \\
$\mathrm{~S}(\mathrm{ppm})$ & 175.80 \\
\hline
\end{tabular}

Fuente: Universidad de los Llanos, Laboratorio de suelos; 2009

\section{DISCUSIÓN}

El análisis de los resultados obtenidos en el proceso de la captura de los gases de combustión de la cascarilla de arroz con soluciones alcalinas de hidróxido de sodio, se refiere a los siguientes aspectos: la disponibilidad del hidróxido del metal alcalino en el medio de la reacción, la precipitación, el rendimiento y la composición química del producto obtenido.

\section{La disponibilidad del hidróxido del metal alcalino en el medio de la reacción}

En el presente trabajo se parte del hecho establecido que para la captura del dióxido de carbono, principal componente de los gases de combustión de la cascarilla de arroz, con soluciones de metales alcalinos o alcalinotérreos en agua, se requiere que la presencia del metal sea suficiente para propiciar la reacción, la que se puede representar con las ecuaciones siguientes para metales alcalinos y alcalinotérreos respectivamente.

$$
\begin{aligned}
& 2 \mathrm{Me}(\mathrm{OH})+\mathrm{CO}_{2}=\mathrm{Me}_{2} \mathrm{CO}_{3}+\mathrm{H}_{2} \mathrm{O} \\
& \mathrm{Me}(\mathrm{OH})_{2}+\mathrm{CO}_{2}=\mathrm{MeCO}_{3}+\mathrm{H}_{2} \mathrm{O}
\end{aligned}
$$

La reacción es efectiva, siempre y cuando el carbonato del metal $\left(\mathrm{Me}_{2} \mathrm{CO}_{3}\right.$ ó $\left.\mathrm{MeCO}_{3}\right)$, se precipite con facilidad por su baja solubilidad en agua y se pueda separar del medio reaccionante. En estas circunstancias el proceso, podría ser un proceso sencillo, de relativa fácil organización.

De los carbonatos conocidos, el carbonato de calcio es de los más insolubles en agua (EI Calcio, 1963). Por tanto, la captura del bióxido de carbono con hidróxido de calcio en solución se presenta como una alternativa importante, además, porque los líquidos y las soluciones, en general, pueden servir como trampas para la captura de gases, pero también, porque los gases de combustión presentan características ácidas $y$, en consecuencia, debe presentarse, en solución acuosa, una reacción ácido - base, lo que lleva a la precipitación y facilita la separación de la sal obtenida.

La disponibilidad del metal en la solución la determina la solubilidad del hidróxido en agua, por esta razón, la baja solubilidad del hidróxido de calcio en agua se convierte en obstáculo para utilizarlo como agente que permita la captura de $\mathrm{CO}_{2}$, puesto que para disponer de las cantidades estequiométricamente necesarias, se requiere volúmenes altos de agua y, por tanto, recipientes de gran tamaño, lo que dificulta la organización del proceso desde el punto de vista práctico. 
En estas circunstancias, se hace necesario encontrar otras fuentes que en lo posible permitan la formación de carbonato de calcio, como las sales de calcio que presenten mayor solubilidad en agua que el hidróxido de este metal alcalinotérreo.

Los ensayos realizados con cloruro de Calcio $\left(\mathrm{CaCl}_{2}\right)$, sal de elevada solubilidad en agua, muestran rendimiento no significativo, presumiblemente por las siguientes razones (Calcio, 1963):

El contacto de la solución de cloruro de Calcio con el $\mathrm{CO}_{2}$ debe llevar a la formación del carbonato de calcio

$\mathrm{CaCl}_{2}+\mathrm{CO}_{2}+\mathrm{H}_{2} \mathrm{O}=\mathrm{CaCO}_{3}+2 \mathrm{HCl}$

Sin embargo, en las condiciones de los ensayos realizados, el carbonato de calcio pudo haberse transformado en bicarbonato (de calcio), de conformidad con la ecuación siguiente, puesto que el flujo de $\mathrm{CO}_{2}$ al medio de la reacción, es constante, (El calcio,1963):

$\mathrm{CaCO}_{3}+\mathrm{CO}_{2}+\mathrm{H}_{2} \mathrm{O}=\mathrm{Ca}\left(\mathrm{HCO}_{3}\right)_{2}$

El bicarbonato de calcio es una sal de elevada solubilidad en agua, que sólo se conoce en solución (El cacio, 1963), por tanto su separación del medio de la reacción no es posible. Sólo queda como alternativa el calentamiento de la solución obtenida, pero esto lleva a desprendimiento de dióxido de carbono, hecho que va en contravía de los objetivos del presente trabajo, a pesar que cierta cantidad de carbonato de calcio se precipita.

$\mathrm{Ca}\left(\mathrm{HCO}_{3}\right)_{2} \stackrel{\mathrm{t}^{\circ} \mathrm{C}}{\longrightarrow} \mathrm{CaCO}_{3}+\mathrm{CO}_{2}+\mathrm{H}_{2} \mathrm{O}$

Ante estos hechos, se hace necesario utilizar hidróxido de sodio $(\mathrm{NaOH})$, de costos, comparativamente, bajos, de solubilidad considerable en agua, para disponer de de las cantidades requeridas del metal alcalino en solución, en recipientes de baja capacidad, que lleve a la posible captura de los gases de combustión, de conformidad con la siguiente ecuación:

$2 \mathrm{NaOH}+\mathrm{CO}_{2}=\mathrm{Na}_{2} \mathrm{CO}_{3}+\mathrm{H}_{2} \mathrm{O}$

El carbonato de sodio $\left(\mathrm{Na}_{2} \mathrm{CO}_{3}\right)$ es soluble en agua, por lo tanto no se precipita en el medio de la reacción, en consecuencia se requiere de etapa adicionales para la precipitación proyectada del carbonato de calcio $\left(\mathrm{CaCO}_{3}\right)$.

El control del proceso de captura de $\mathrm{CO}_{2}$, con soluciones acuosas de $\mathrm{NaOH}$, de conformidad con los resultados expuestos en la Tabla 2, es una actividad relativamente sencilla, en la que de indicador puede servir el valor del pH de la solución, con valores del orden de 12 unidades al iniciar el proceso y de 8,3 - al concluirlo, dados los aceptables coeficientes de variación obtenidos (Tabla 2).

Por el contrario, la duración del proceso fluctúa, de manera sensible (Tabla 1), evento que debe tener relación la estructura de la cascarilla que no ofrece igual disponibilidad del carbono, dentro de élla, para generar la combustión al contacto con el oxígeno (Arcos, 2007), como también por la diferentes condiciones climáticas en los días de realización del experimento, en especial la humedad relativa, puesto que la humedad de la cascarilla con la que se trabajó, fue inferior al $8 \%$, en todos los casos.

La precipitación, el rendimiento y la composición química del carbonato de calcio obtenido

La precipitación del carbonato de calcio al tratar la solución de carbonato de sodio, obtenido en la etapa anterior, con una solución de cloruro de calcio, se logra de conformidad con la siguiente ecuación: $\mathrm{Na}_{2} \mathrm{CO}_{3}+\mathrm{CaCl}_{2}=\mathrm{CaCO}_{3} \downarrow+2 \mathrm{NaCl}$;

El carbonato de calcio se precipita y el cloruro de sodio se incorpora a la solución acuosa, cuyo volumen se aproxima a los 10 litros de la solución inicial. 
Para el cálculo del rendimiento del carbonato de calcio se tuvo en cuenta la masa (100 gramos) de hidróxido de sodio utilizado en la captura del dióxido de carbono, generado por combustión de la cascarilla, en calidad de reactivo límite, en razón que la masa de cascarilla puede ser considerada en exceso, de conformidad con la Ecuación siguiente:

$$
2 \mathrm{NaOH}+\mathrm{CO}_{2}=\mathrm{Na}_{2} \mathrm{CO}_{3}+\mathrm{H}_{2} \mathrm{O}
$$

Con base en los datos reportados por el Laboratorio de Microscopia Electrónica de la Universidad Nacional, sede Medellín, UNALMED, sobre la composición química de la cascarilla de arroz cruda, el porcentaje mínimo de carbono podría ser del orden de $14.8 \%$, por tanto, $4 \mathrm{~kg}$ de cascarilla utilizados, en cada ensayo, contendrían $592 \mathrm{~g}$ de carbono, los cuales, de conformidad con la Ecuación 1, generarían $2.170 \mathrm{~g}$ de $\mathrm{CO}_{2}$, que a su vez requerirían $3.946 \mathrm{~g}$ de $\mathrm{NaOH}$ (ecuación 11); para producir $3.790 \mathrm{~g}$ de $\mathrm{CaCO}_{3}$.

Teniendo en cuenta que se utilizaron sólo $100 \mathrm{~g}$ de $\mathrm{NaOH}$, estos deben reaccionar con $55 \mathrm{~g}$ de $\mathrm{CO}_{2}$, para generar $133 \mathrm{~g}$ de $\mathrm{Na}_{2} \mathrm{CO}_{3}$ y obtener $125 \mathrm{~g}$ de $\mathrm{CaCO}_{3}$. Puesto que la masa de carbonato de calcio $\left(\mathrm{CaCO}_{3}\right.$ ) obtenido es en promedio de $66,4 \mathrm{~g}$, se puede aceptar que el rendimiento del proceso, dentro de estas consideraciones, es del orden del $53 \%$.

Es claro que dentro de estos criterios de evaluación no se tiene en cuenta la totalidad del carbono presente en la muestra de cascarilla

\section{CONCLUSIONES}

Los gases de combustión de la cascarilla de arroz, entre ellos el dióxido de carbono $\left(\mathrm{CO}_{2}\right)$, pueden ser capturados con soluciones alcalinas de hidróxido de potasio $(\mathrm{KOH})$ e hidróxido de sodio $(\mathrm{NaOH})$.

El reducción del pH de la solución alcalina, al iniciar y concluir el proceso, puede servir de indicador de captura de los gases de combustión.

El producto de la captura de los gases de combustión con soluciones alcalinas puede ser utilizada. Sin embargo este análisis se adelantará en investigaciones futuras, puesto que la finalidad del presente trabajo consistía en demostrar la posibilidad de capturar el $\mathrm{CO}_{2}$ generado en procesos de combustión con soluciones alcalinas, situación que puede considerarse superada y evidenciada claramente por los resultados de la composición química del producto obtenido, expuestos en la Figura 3.

\section{Elementos para el aprovechamiento integral de la cascarilla de arroz}

Los resultados obtenidos en el presente trabajo, que aunque tiene las características de preliminar, permiten considerar posible la captura de los gases de combustión de la cascarilla de arroz, en particular el bióxido de carbono con soluciones de metales alcalinos y su posterior conversión en carbonato de calcio de amplio uso en la producción agrícola.

En estas condiciones, podría plantearse la alternativa de utilizar la descomposición térmica de la cascarilla de arroz como una alternativa de aprovechamiento integral de este abundante residuo de la producción de zonas arroceras. Puesto que el calor que se genera en el proceso puede ser y es aprovechado en procesos agroindustriales y de alimentos que lo requieran, las cenizas y el carbonato de calcio que se obtiene a partir de la captura de los gases de combustión puede aplicarse en cultivos agrícolas y las aguas con contenidos de cloruros - en riego.

transformado en carbonato de calcio $\left(\mathrm{CaCO}_{3}\right)$ por precipitación con soluciones acuosas de cloruro de calcio $\left(\mathrm{CaCl}_{2}\right)$.

El producto básico de la captura de los gases de combustión con soluciones alcalinas y su posterior transformación es carbonato de Calcio que puede ser aprovechado con fines agrícolas.

La composición química de las cenizas obtenidas durante el proceso de combustión, indica que estas pueden ser de gran utilidad en actividades 
agrícolas; en especial si se emplean como fuente de elementos de fosforo, manganeso y azufre.

Los resultados obtenidos en el presente trabajo permiten visualizar la posibilidad de utilizar la descomposición térmica de la cascarilla de arroz

\section{RECOMENDACIONES}

Cuantificar el contenido de dióxido de carbono $\left(\mathrm{CO}_{2}\right)$ generado durante el proceso de combustión, ya que este gas es un indicador directo del rendimiento en la obtención de carbonato de calcio $\left(\mathrm{CaCO}_{3}\right)$ en la captura de los gases de combustión proceso.

Optimizar la captura de dióxido de carbono $\left(\mathrm{CO}_{2}\right)$ en el proceso de combustión para lograr mayor

\section{REFERENCIAS}

Ajnazarova L, Kafarov V. Optimización de experimentos en la ciencia y tecnología químicas. Moscú. Vyschaya Shkola. 1978. 215 p. En ruso.

Arcos A. et al. La cascarilla de arroz como fuente de $\mathrm{SiO}_{2}$ Rev. Fac. Ing. Univ. Antioquia. 2007; 41: 7-20.

Calleja J. "Las Puzolanas", Separata de la Revista ION Vols. XXIX y XXX, Madrid, 1968.

Canadell JG, Le Que'ré C, Raupach MR, Field CB, Buitenhuis ET, Ciais P, et al. Contributions to accelerating atmospheric $\mathrm{CO}_{2}$ growth from economic activity, carbon intensity and efficiency of natural sinks. Proc.Natl. Acad. Sci. 2007; 104(47): 1886618870.

Castellanos M. Adsorción de sustancias e iones metálicos en aguas residuales empleando carbón activado obtenido de meollo. Tesis para optar al Título de PhD. Instituto D.I. Mendeleyev de Moscú. 1984.

Cortés K. Estudio de la descomposición microbiológica de la cascarilla de arroz. Trabajo de Grado para optar al título de Ingeniero Agrónomo. Unillanos.1999. como una alternativa para su aprovechamiento integral, en razón que el calor, las cenizas, el carbonato de calcio producto de la captura de los gases de combustión y las aguas efluentes, ricas en cloruros, pueden ser aprovechadas en procesos productivos.

rendimiento en la obtención de carbonato de calcio $\left(\mathrm{CaCO}_{3}\right)$ con relación al contenido de carbono en la cascarilla de arroz.

Precisar el tiempo de combustión de la cascarilla como parámetro básico en aplicaciones de la alternativa planteada en mayor escala.

Degremont. 1979. Manual técnico del agua. Cuarta edición, España.

El calcio. Enciclopedia química.1963. Moskva. P.370-383. En ruso.

Federación Nacional de Arroceros. Producción de arroz paddy seco en Colombia por zonas desde 2000 hasta 2010. Informacion estadística. FNA. 2011.En:http://www.fedearroz.com.co/ apr_public.php consultado abril 142010.

Fernández A. Aspectos Industriales de la Producción de Carbón Activado y sus Aplicaciones en la Mitigación Ambiental. Congreso Iberoamericano de Metalurgia y Materiales, Habana, Cuba, Octubre 8 de 2006.

Forero L. Alternativas de uso de la cascarilla de arroz. En:http://www.andi.com.co/Camaras/ Induarroz/PaginasNuevas/Default21.htm

Flores Carlos, composición del cemento, Universidad nacional de Cajamarca, facultad de ingeniería 2004 disponible en http://www. Scribd.com/go/24863679composicion-quimica-delcemento 
Gorachategi M. Seguridad alimentaria: dioxinas. XVII Curso de Especialización FEDNA, 2001. EN. http://www.etsia.upm.es/fedna/capitulos/ 2001CAPVIII.pdf

Hernández G. El efecto de invernadero. 2001. En: http://www.aie.org.ar/downloads/invernadero.pdf

Krishnarao RV, Subrahmanyam J, Jagadish Kumar T. Studies on the Formation of Black Particles in Rice Husk Silica Ash. Journal of the European Ceramic Society. 2001. Vol. 21:99-104. Comentado en: Martínez JD, Pineda TG, López JP, Betancur $M$ Experimentos de combustión con cascarilla de arroz en lecho fluidizado para la producción de ceniza rica en sílice Rev. Fac. Ing. Univ. Antioquia. 2010; 51: 97-104.

Jodakov V. Química inorgánica. Moscú 1977.

La Cadena del Arroz en Colombia. Una mirada global de su estructura y dinámica, 1991-2005. Ministerio de Agricultura y Desarrollo Rural. Observatorio Agrocadenas Colombia. Bogotá, Marzo de 2005. Disponible en internet en: www.agronet.gov.co/www/docs_agronet/ 2005112141728_caracterizacion_arroz.pdf

Labrada B, Lago CM, Marzal N, Salas D, Estudio de la pirólisis discontinua del bagazo de caña Universidad de Oriente. Tecnología química. 1998; 18(1-2):65.

Lackner KS, Grimes P, Ziock HJ. Capturing carbon dioxide from air. In: $24^{\text {th }}$ Annual Technical Conference on Coal Utilization, 1999. Clearwater FL.

Li Y, Zhao C, Ren Q, Duan L, Chen H, Chen X. Effect of rice husk ash addition on $\mathrm{CO}_{2}$ capture behavior of calcium-based sorbent during calcium looping cycle. Fuel Processing Technology. 2009; 90:825-834.

López G. Futuro energético de Costa Rica. 2006. Disponible en internet en: http://www.cyted.org/ Fibecyt/forumcytediberoeka2006/Documentacion/ Resumenes\%20de\%20Ponencias/Sesion\%202.1/ 2.1\%20Guillermo\%20Lopez\%20(Costa\%20Rica).pdf
Mahmoudkhani M, Keith DW. Low-energy sodium hydroxide recovery for $\mathrm{CO}_{2}$ capture from atmospheric air-Thermodynamic analysis. Int. J. Greenhouse Gas Control 2009, doi:10.1016/ j.ijggc.2009.02.003

Martínez JD, Pineda TG, López JP, Betancur M Experimentos de combustión con cascarilla de arroz en lecho fluidizado para la producción de ceniza rica en sílice Rev. Fac. Ing. Univ. Antioquia. 2010; 51: 97-104.

Melnik B. Manual de ingeniería. Tecnología de la sustancias inorgánicas, 1975. Jimia, Moscú. En ruso.

Nikulshina V, Gálvez ME, Steinfeld A. Kinetic analysis of the carbonation reactions for the capture of $\mathrm{CO}_{2}$ from air via the $\mathrm{Ca}(\mathrm{OH})_{2}-\mathrm{CaCO}_{3}-\mathrm{CaO}$ solar thermochemical cycle. Chemical Engineering Journal. 2007; 129: 75-83.

Pérez, E. Estudio teórico experimental de la fluidodinámica del proceso de gasificación en lecho fluidizado de bagazo de caña. Técnicas de energía renovable. Tesis de Maestría. Universidad Internacional de Andalucía. Sede iberoamericana La Rábida. Santa Clara, Cuba, 1997.

Prada A. Elaboración de métodos para la eliminación de sustancias de hiero y manganeso del agua. Tesis para optar al Título de PhD. Instituto D.I. Mendeleyev de Moscú. 1986.

Reactivos y Productos químicos. MERCK. Bogotá. Colombia. 2007.

Spector NA, Dodge BF. Removal of carbon dioxide from atmospheric air. Trans. Am. Inst. Chem. Eng. 1946. 42]: 827-848. Comentado en: Mahmoudkhani M, Keith DW. Low-energy sodium hydroxide recovery for $\mathrm{CO} 2$ capture from atmospheric air-Thermodynamic analysis. Int. J. Greenhouse Gas Control 2009, doi:10.1016/j.ijggc.2009.02.003

Tardieu J, De La Torriente A. Aprovechamiento energético de los subproductos del cultivo del arroz. AEPO. 2003 Departamento de energía e 
instalaciones. En: http://www.agroinformacion.com/ leer-articulo.aspx?not=219

Tepe JB, Dodge BF. Absorption of carbon dioxide by sodium hydroxide solutions in a packed column. Trans. Am. Inst. Chem. Eng. 1943. 39: 255276 Comentado en: Mahmoudkhani M, Keith DW. Low-energy sodium hydroxide recovery for $\mathrm{CO} 2$ capture from atmospheric air-Thermodynamic analysis. Int. J. Greenhouse Gas Control 2009, doi:10.1016/j.jjggc.2009.02.003
Vaidya PD, Konduru P, Vaidyanathan M, Kenig EY. Kinetics of Carbon Dioxide Removal by Aqueous Alkaline Amino Acid Salts. Industrial \& Engineering Chemistry Research 2010; 49(21):11067-11072.

Varón CJ. Diseño, construcción y puesta a punto de un prototipo de quemador para la combustión continua y eficiente de la cascarilla de arroz. El Hombre y la Máquina No. 25. Julio - Diciembre de 2005. 